\title{
Nonlinear dielectric relaxation and dynamic Kerr effect in a strong dc electric field suddenly switched on: Exact solutions for the three-dimensional rotational diffusion model
}

\author{
W. T. Coffey \\ Department of Electronic and Electrical Engineering, Trinity College, Dublin 2, Ireland \\ J. L. Déjardin* \\ Centre d'Etudes Fondamentales, Université de Perpignan, 52 Avenue de Villeneuve, 66860 Perpignan Cedex, France \\ Yu. P. Kalmykov and S. V. Titov \\ Institute of Radio Engineering and Electronics of the Russian Academy of Sciences, Vvedenskii Square 1, \\ Fryazino, Moscow Region, 141120, Russian Federation
}

(Received 8 July 1996)

\begin{abstract}
The infinite hierarchy of differential-recurrence relations for ensemble averages of the spherical harmonics pertaining to the noninertial rotational Brownian motion of an ensemble of polar and anisotropically polarizable molecules in a strong external dc electric field is derived by averaging the underlying Langevin equation. This procedure avoids recourse to the Fokker-Planck equation, the solution of which involves complicated mathematical manipulations. Exact analytic solutions for the spectra of the relaxation functions and relaxation times for nonlinear dielectric relaxation and dynamic Kerr effect of symmetric top molecules are calculated for two limiting cases, namely, pure induced dipole moments and pure permanent moments, using the continued fraction method. The general case where both types of moment are taken into account is then considered by using matrix continued fractions. Exact expressions for the dielectric and Kerr effect relaxation times are also derived as functions of the parameters $\xi$ and $\sigma$ characterizing the field-off and the induced dipole moments. Plots of these relaxation times are presented for various values of $\xi$ and $\sigma$. The nonlinear relaxation behavior is emphasized in figures showing how the real and imaginary parts of the spectra of the relaxation functions deviate from the Lorentzian profiles. [S1063-651X(96)12512-5]

PACS number(s): 42.70.Df, 05.40.+j, 78.20.Fm, 78.20.Jq
\end{abstract}

\section{INTRODUCTION}

Dielectric and Kerr effect relaxation of polar fluids springs from the rotational motion of molecules in the presence of external electric fields and thermal agitation (see, e.g., [1-3]). Interpretation of these phenomena is usually based on the rotational diffusion model in the noninertial limit which relies on the solution of the appropriate FokkerPlanck equation and has usually been confined to the linear response or the nonlinear response in low order of perturbation theory [4], where the energy of a molecule in the electric field is far less than the thermal energy. This restricts considerably the range of the applicability of the theory.

The theoretical approach to the analysis of nonlinear dielectric and Kerr effect relaxation experiments when inertial effects are neglected usually starts with the underlying Langevin equation whence the Fokker-Planck equation for the probability distribution function $W(\{\mathbf{u}\}, t)$ of orientations of a unit vector $\mathbf{u}$ fixed in the particle in configuration space [5] is derived with the aid of the continuity equation

$$
\frac{\partial}{\partial t} W+\operatorname{div}(\dot{\mathbf{u}} W)=0
$$

which is the probability conservation law. The FokkerPlanck equation can then be solved by expanding the distri-

\footnotetext{
*Author to whom correspondence should be addressed.
}

bution function $W(\{\mathbf{u}\}, t)$ as a series of spherical harmonics. This yields an infinite hierarchy of linear differentialrecurrence equations for averaged spherical harmonics. The general method of solution of this hierarchy is effected by successively increasing the number of equations until convergence is attained. An alternative solution may be formulated by using continued fractions [5-7]. Numerical and approximate methods for solving the Fokker-Planck equation have been discussed elsewhere (see, for example, Refs. [5-10]).

The goal of the present paper is to derive exact analytic equations for the dielectric and Kerr effect response functions and relaxation times when a strong static electric field is suddenly applied to an assembly of noninteracting polar and anisotropically polarizable molecules. For this purpose we shall apply an analytical method recently developed for the calculation of the linear response of systems of particles compelled to rotate in three-dimensional space [7,10-14]. The essence of this method is the exact analytical solution of the infinite hierarchy of differential-recurrence relations governing the relaxation dynamics of a Brownian particle in the presence of an external potential by means of ordinary or matrix continued fractions. The method also constitutes a particularly simple way of deriving the hierarchy of linear differential-recurrence relations for desired averages for any particular external potential from the vector nonlinear EulerLangevin equation for a polar molecule. It is often very difficult to obtain such a hierarchy from the Fokker-Planck equation because of the problems involved in separating the 
variables. This eliminates the excessive step in the theory of constructing and solving the corresponding Fokker-Planck equation entirely. In Refs. [7,9-14] we have demonstrated using several physical examples the applicability of the method to the calculation of generalized complex susceptibilities and correlation times for dynamic variables governed by nonlinear Langevin equations in the linear response case. In the present paper we apply this method to evaluate the nonlinear response for dielectric and Kerr effect relaxation. Our method just as that of Watanabe and Morita [6] is based on continued fractions. However, in contrast to the approach of Ref. [6], it allows us to obtain solutions of inhomogeneous recurrence equations (which are necessary for calculating the relaxation times). Furthermore, it can be applied to the general case of polar and polarizable molecules, rather than to the particular cases of nonpolar and/or nonpolarizable molecules. Déjardin, Blaise, and Coffey [15] have demonstrated recently the applicability of the method to the calculation of the transient birefringence due to the induced dipole Kerr effect only. Here we consider the general case of polar and anisotropically polarizable molecules, the nonlinear dielectric and Kerr effect relaxation being considered simultaneously. We remark that these problems, unlike the ones considered in Refs. [7,9-14], are truly nonlinear; therefore, there is no longer any connection between the step-on response and the ac response and so the concept of relaxation functions and relaxation times should be used rather than correlation functions and correlation times.

\section{ROTATIONAL DIFFUSION IN A STRONG ELECTRIC FIELD AND RELATED RELAXATION FUNCTIONS}

We study the three-dimensional rotational Brownian motion of a particle in an external electric field. The particle contains a rigid electric dipole $\boldsymbol{\mu}$. We take a unit vector $\mathbf{u}(t)$ through the center of mass of the particle in the direction of $\boldsymbol{\mu}$. Then the rate of change of $\mathbf{u}(t)$ is

$$
\frac{d \mathbf{u}(t)}{d t}=\boldsymbol{\omega}(t) \times \mathbf{u}(t),
$$

where $\boldsymbol{\omega}(t)$ is the angular velocity of the particle. It should be noted that Eq. (2.1) is a purely kinematic relation with no particular reference either to the Brownian movement or to the shape of the particle. For simplicity we specialize it to the rotational Brownian motion of a symmetrical top molecule by supposing that the angular velocity $\boldsymbol{\omega}(t)$ obeys the Euler-Langevin equation [7]

$$
\hat{I} \frac{d \boldsymbol{\omega}(t)}{d t}+\zeta \boldsymbol{\omega}(t)=\mathbf{m}(t) \times \mathbf{E}(t)+\boldsymbol{\lambda}(t),
$$

where $\hat{I}$ is the inertia tensor of the molecule, $\mathbf{m}$ is the total dipole moment which we represent as

$$
\mathbf{m}(t)=\boldsymbol{\mu}(t)+\hat{\alpha}(t) \cdot \mathbf{E}(t),
$$

$\hat{\alpha}$ is the molecular polarizability tensor (thus effects due to the hyperpolarizability are neglected), $\zeta \boldsymbol{\omega}(t)$ is the damping torque due to Brownian movement, and $\boldsymbol{\lambda}(t)$ is the white- noise-driving torque, again due to Brownian movement so that $\boldsymbol{\lambda}(t)$ has the following properties:

$$
\begin{gathered}
\overline{\lambda_{i}(t)}=0, \\
\overline{\lambda_{i}\left(t_{1}\right) \lambda_{j}\left(t_{2}\right)}=2 k T \zeta \delta_{i j} \delta\left(t_{1}-t_{2}\right) .
\end{gathered}
$$

The overbar means a statistical average over an ensemble of molecules which all start at time $t$ with the same angular velocity $\boldsymbol{\omega}$ and orientation $\mathbf{u}$ (sharp initial conditions) [5,7], $\delta_{i j}$ is Kronecker's delta, $i, j=1,2,3$, which correspond to the Cartesian axes $x, y, z$ of the fixed coordinate system, $\delta(t)$ is the Dirac delta function. The term $\mathbf{m}(t) \times \mathbf{E}(t)$ in Eq. (2.2), is the torque due to the total electric field acting on the molecule. This torque can be expressed in terms of the potential function $V(\{\mathbf{u}\})$ as a function of the components of the vector $\mathbf{u}$,

$$
\mathbf{m} \times \mathbf{E}=-\mathbf{u} \times \frac{\partial}{\partial \mathbf{u}} V(\{\mathbf{u}\}),
$$

where

$$
\frac{\partial}{\partial \mathbf{u}}=\mathbf{i} \frac{\partial}{\partial u_{x}}+\mathbf{j} \frac{\partial}{\partial u_{y}}+\mathbf{k} \frac{\partial}{\partial u_{z}} .
$$

$\mathbf{i}, \mathbf{j}$, and $\mathbf{k}$ are the unit vectors along the Cartesian axes $x, y$, and $z$, respectively; $u_{x}, u_{y}$, and $u_{z}$ are the Cartesian components of the unit vector $\mathbf{u}(t)$. These components are expressed in terms of the polar $(\vartheta)$ and azimuthal $(\varphi)$ angles as follows:

$$
u_{x}=\sin \vartheta \cos \varphi, \quad u_{y}=\sin \vartheta \sin \varphi, \quad u_{z}=\cos \vartheta
$$

In the simplest case of a symmetric top molecule with the field $\mathbf{E}(t)$ applied along the $z$ axis the potential function $V(\{\mathbf{u}\})$ is given by [6]

$$
V(\{\mathbf{u}\}, t)=-\mu E(t) \cos \vartheta-\frac{1}{2}\left(\alpha_{1}-\alpha_{2}\right) E^{2}(t) \cos ^{2} \vartheta,
$$

where $\alpha_{1}$ and $\alpha_{2}$ are the components of the electric polarizability parallel and perpendicular to the axis of symmetry of the molecule.

Equation (2.2) includes the inertia of the molecule. The noninertial or low-frequency response (the Debye approximation) occurs when we neglect the inertia term in Eq. (2.2). In this limit the angular velocity vector may be immediately obtained from Eq. (2.2) as

$$
\boldsymbol{\omega}(t)=[\mathbf{m}(t) \times \mathbf{E}(t)+\boldsymbol{\lambda}(t)] \zeta^{-1} .
$$

On combining this equation with the kinematic relation Eq. (2.1) one obtains

$$
\frac{d \mathbf{u}(t)}{d t}=-\zeta^{-1}\left[\mathbf{u}(t) \times \frac{\partial}{\partial \mathbf{u}} V\right] \times \mathbf{u}(t)+\zeta^{-1} \boldsymbol{\lambda}(t) \times \mathbf{u}(t),
$$

which, using the properties of the triple vector product, becomes 
$\frac{d \mathbf{u}(t)}{d t}=-\zeta^{-1}\left[\frac{\partial}{\partial \mathbf{u}} V-\mathbf{u}(t)\left(\mathbf{u}(t) \cdot \frac{\partial}{\partial \mathbf{u}} V\right)\right]+\zeta^{-1} \boldsymbol{\lambda}(t) \times \mathbf{u}(t)$.

This is the vector Langevin equation for the motion of the vector $\mathbf{u}$ in the noninertial limit. Equation (2.6) is equivalent to three equations for the Cartesian components of $\mathbf{u}$

$$
\begin{aligned}
\frac{d}{d t} u_{x}(t)= & \zeta^{-1}\left[\lambda_{y}(t) u_{z}(t)-\lambda_{z}(t) u_{y}(t)-\frac{\partial}{\partial u_{x}} V\right. \\
& \left.+u_{x}\left(\mathbf{u}(t) \cdot \frac{\partial}{\partial u} V\right)\right], \\
\frac{d}{d t} u_{y}(t)= & \zeta^{-1}\left[\lambda_{z}(t) u_{x}(t)-\lambda_{x}(t) u_{z}(t)-\frac{\partial}{\partial u_{y}} V\right. \\
& \left.+u_{y}\left(\mathbf{u}(t) \cdot \frac{\partial}{\partial \mathbf{u}} V\right)\right] \\
\frac{d}{d t} u_{z}(t)= & \zeta^{-1}\left[\lambda_{x}(t) u_{y}(t)-\lambda_{y}(t) u_{x}(t)-\frac{\partial}{\partial u_{z}} V\right. \\
& \left.+u_{z}\left(\mathbf{u}(t) \cdot \frac{\partial}{\partial \mathbf{u}} V\right)\right] .
\end{aligned}
$$

The stochastic differential equations (2.7)-(2.9) contain multiplicative noise terms $\lambda_{i}(t) u_{j}(t)$. This poses an interpretation problem for these equations as has been discussed in Refs. [5, 7]. We recall, taking the Langevin equation for $N$ stochastic variables $\{\boldsymbol{\xi}(t)\}=\left\{\xi_{1}(t), \xi_{2}(t), \ldots, \xi_{N}(t)\right\}$ :

$$
\frac{d \xi_{i}(t)}{d t}=h_{i}(\{\boldsymbol{\xi}(t)\}, t)+g_{i j}(\{\boldsymbol{\xi}(t)\}, t) \Gamma_{j}(t)
$$

with

$$
\begin{gathered}
\overline{\Gamma_{i}(t)}=0, \\
\overline{\Gamma_{i}\left(t_{1}\right) \Gamma_{j}\left(t_{2}\right)}=2 D \delta_{i j} \delta\left(t_{1}-t_{2}\right)
\end{gathered}
$$

and interpreting it as a Stratonovich equation, that the averaged equation for the sharp values $\xi_{i}(t)=x_{i}$ at time $t$ is $[5,7]$

$$
\begin{aligned}
\frac{d x_{i}}{d t} & =\lim _{\tau \rightarrow 0} \frac{\overline{\xi_{i}(t+\tau)-x_{i}}}{\tau} \\
& =h_{i}(\{\mathbf{x}\}, t)+D g_{k j}(\{\mathbf{x}\}, t) \frac{\partial}{\partial x_{k}} g_{i j}(\{\mathbf{x}\}, t),
\end{aligned}
$$

where $\xi_{i}(t+\tau)(\tau>0)$ is the solution of Eq. (2.10) with the initial conditions $\xi_{i}(t)=x_{i}$. In Eqs. (2.10) and (2.12) the summation over $j$ and $k$ is understood (Einstein's notation). The last term in Eq. (2.12) is called the noise-induced or spurious drift [7]. The proof of Eq. (2.12) can be found elsewhere (see Ref. [5], pp. 54, 55).

In the same manner we can prove that the averaged equation for an arbitrary differentiable function $f(\{\boldsymbol{\xi}\})$ has the following form (see the Appendix):

$$
\begin{aligned}
\frac{d f(\{\mathbf{x}\})}{d t}= & h_{i}(\{\mathbf{x}\}, t) \frac{\partial}{\partial x_{i}} f(\{\mathbf{x}\})+D g_{k j}(\{\mathbf{x}\}, t) \\
& \times \frac{\partial}{\partial x_{k}}\left[g_{i j}(\{\mathbf{x}\}, t) \frac{\partial}{\partial x_{i}} f(\{\mathbf{x}\})\right],
\end{aligned}
$$

where summation over $i, j$, and $k$ is also understood.

We remark that we shall always use the Stratonovich definition [5,7] of the average of the multiplicative noise term here as that definition always constitutes the mathematical idealization of the physical stochastic process of orientational relaxation in the noninertial limit. Thus, it is unnecessary to transform the Langevin equations (2.7)-(2.9) to Itô equations (e.g., [18]). Moreover, we can apply the methods of ordinary analysis $[5,18]$.

In the study of orientation relaxation the quantities of interest are the spherical harmonics $X_{n m}$ defined as

$$
\begin{aligned}
X_{n \pm m} & =e^{ \pm i m \varphi} P_{n}^{m}(\cos \vartheta) \\
& =e^{ \pm i m \varphi}\left(1-\cos ^{2} \vartheta\right)^{m / 2} \frac{d^{m} P_{n}(\cos \vartheta)}{d \cos \vartheta^{m}},
\end{aligned}
$$

where $P_{n}(x)$ and $P_{n}^{m}(x)$ are the Legendre polynomials and the associated Legendre functions, respectively [19]. The $X_{n \pm m}$ are expressed in terms of $u_{x}, u_{y}, u_{z}$ as follows:

$$
X_{n \pm m}=\left(u_{x} \pm i u_{y}\right)^{m} \frac{d^{m} P_{n}\left(u_{z}\right)}{d u_{z}^{m}} .
$$

Noting that according to the Stratonovich definition the conventional rules of transformation of a stochastic variable can be used [18] and that

$$
\begin{aligned}
\frac{d}{d t} X_{n m}= & m\left(u_{x}+i u_{y}\right)^{m-1} \frac{d^{m} P_{n}\left(u_{z}\right)}{d u_{z}^{m}} \frac{d}{d t} u_{x} \\
& +i m\left(u_{x}+i u_{y}\right)^{m-1} \frac{d^{m} P_{n}\left(u_{z}\right)}{d u_{z}^{m}} \frac{d}{d t} u_{y} \\
& +\left(u_{x}+i u_{y}\right)^{m} \frac{d^{m+1} P_{n}\left(u_{z}\right)}{d u_{z}^{m+1}} \frac{d}{d t} u_{z}
\end{aligned}
$$

we can obtain the equation of motion of the spherical harmonics $X_{n m}$ by cross-multiplying Eqs. (2.7)-(2.9) by

$$
\begin{gathered}
m\left(u_{x}+i u_{y}\right)^{m-1} \frac{d^{m} P_{n}\left(u_{z}\right)}{d u_{z}^{m}}, \\
i m\left(u_{x}+i u_{y}\right)^{m-1} \frac{d^{m} P_{n}\left(u_{z}\right)}{d u_{z}^{m}}, \\
\left(u_{x}+i u_{y}\right)^{m} \frac{d^{m+1} P_{n}\left(u_{z}\right)}{d u_{z}^{m+1}},
\end{gathered}
$$

respectively, and then summing them. Thus, 


$$
\begin{aligned}
\frac{d}{d t} X_{n m}(\{\mathbf{u}(t)\})= & \frac{1}{\zeta}\left\{\left[u_{x}(t)+i u_{y}(t)\right]^{m} \frac{d^{m+1} P_{n}\left[u_{z}(t)\right]}{d u_{z}^{m+1}(t)}\left[-\frac{\partial}{\partial u_{z}} V+u_{z}(t)\left(\mathbf{u}(t) \cdot \frac{\partial}{\partial \mathbf{u}} V\right)\right]\right. \\
& \left.+m\left[u_{x}(t)+i u_{y}(t)\right]^{m-1} \frac{d^{m} P_{n}\left[u_{z}(t)\right]}{d u_{z}^{m}(t)}\left[-\frac{\partial}{\partial u_{x}} V-i \frac{\partial}{\partial u_{y}} V+\left[u_{x}(t)+i u_{y}(t)\right]\left(\mathbf{u}(t) \cdot \frac{\partial}{\partial \mathbf{u}} V\right)\right]\right\} \\
& +\left\{m\left[u_{x}(t)+i u_{y}(t)\right]^{m-1} \frac{d^{m} P_{n}\left(u_{z}(t)\right)}{d u_{z}^{m}(t)}\left\{g_{x j}(\{\mathbf{u}(t)\})+i g_{y j}[\{\mathbf{u}(t)\}]\right\}\right. \\
& \left.+\left[u_{x}(t)+i u_{y}(t)\right]^{m} \frac{d^{m+1} P_{n}\left[u_{z}(t)\right]}{d u_{z}^{m+1}(t)} g_{z j}(\{\mathbf{u}(t)\})\right\} \lambda_{j}(t)
\end{aligned}
$$

where the components of the tensor $\mathbf{g}$ are

$$
\begin{aligned}
& g_{x x}=0, \quad g_{x y}=u_{z} / \zeta, \quad g_{x z}=-u_{y} / \zeta, \\
& g_{y x}=-u_{z} / \zeta, \quad g_{y y}=0, \quad g_{y z}=u_{x} / \zeta, \\
& g_{z x}=u_{y} / \zeta, \quad g_{z y}=-u_{x} / \zeta, \quad g_{z z}=0 .
\end{aligned}
$$

On averaging the stochastic equation (2.16) noting Eq. (2.13), we have

$$
\begin{aligned}
2 \tau_{D} \frac{d}{d t} X_{n m}= & \frac{1}{k T}\left\{m\left(u_{x}+i u_{y}\right)^{m-1} \frac{d^{m} P_{n}\left(u_{z}\right)}{d u_{z}^{m}}\left[-\frac{\partial}{\partial u_{x}} V-i \frac{\partial}{\partial u_{y}} V+\left(u_{x}+i u_{y}\right)\left(\mathbf{u} \cdot \frac{\partial}{\partial \mathbf{u}} V\right)\right]\right. \\
& \left.+\left(u_{x}+i u_{y}\right)^{m} \frac{d^{m+1} P_{n}\left(u_{z}\right)}{d u_{z}^{m+1}}\left[-\frac{\partial}{\partial u_{z}} V+u_{z}\left(\mathbf{u} \cdot \frac{\partial}{\partial \mathbf{u}} V\right)\right]\right\} \\
& +\zeta^{2} g_{k j} \frac{\partial}{\partial u_{k}}\left[m\left(u_{x}+i u_{y}\right)^{m-1} \frac{d^{m} P_{n}\left(u_{z}\right)}{d u_{z}^{m}}\left(g_{x j}+i g_{y j}\right)+\left(u_{x}+i u_{y}\right)^{m} \frac{d^{m+1} P_{n}\left(u_{z}\right)}{d u_{z}^{m+1}} g_{z j}\right],
\end{aligned}
$$

where

$$
\tau_{D}=\frac{\zeta}{2 k T}
$$

is the Debye relaxation time. We remark that $u_{x}, u_{y}, u_{z}$ in Eq. (2.18) and $u_{x}(t), u_{y}(t), u_{z}(t)$ in Eqs. (2.7) - (2.9) have different meanings, namely, $u_{x}(t), u_{y}(t), u_{z}(t)$ in Eqs. (2.7)-(2.9) are stochastic variables while $u_{x}, u_{y}, u_{z}$ in Eq. (2.18) are the sharp (definite) values $u_{k}(t)=u_{k}$ at time $t$. Instead of using different symbols for the two quantities we have distinguished sharp values at time $t$ from stochastic variables by deleting the time argument, as in Ref. [7]. The right-hand side of Eq. (2.18) consists of two terms, namely, the deterministic drift and the noise-induced (or spurious) drift. These terms have been evaluated elsewhere $[7,16]$.

The quantities $X_{n m}$ in Eq. (2.18) are in general functions of $u_{k}$, which are themselves random variables with probability density function $W$ such that $W d u_{k}$ is the probability of finding $u_{k}$ in the interval $\left(u_{k}, u_{k}+d u_{k}\right)$. Therefore in order to obtain equations for the moments which govern the relaxation dynamics of the system we must also average Eq. (2.18) over the probability density function $W[8]$. We have

$$
\begin{aligned}
2 \tau_{D} \frac{d}{d t}\left\langle X_{n m}\right\rangle+n(n+1)\left\langle X_{n m}\right\rangle= & \frac{1}{2 k T(2 n+1)}\left[n\left\langle X_{n+1 m+1}\left(\frac{\partial}{\partial u_{x}} V-i \frac{\partial}{\partial u_{y}} V\right)\right\rangle+(n+1)\left\langle X_{n-1 m+1}\left(\frac{\partial}{\partial u_{x}} V-i \frac{\partial}{\partial u_{y}} V\right)\right\rangle\right] \\
& -\frac{1}{2 k T(2 n+1)}\left[n(n-m+1)(n-m+2)\left\langle X_{n+1 m-1}\left(\frac{\partial}{\partial u_{x}} V+i \frac{\partial}{\partial u_{y}} V\right)\right\rangle\right. \\
& \left.+(n+1)(n+m-1)(n+m)\left\langle X_{n-1 m-1}\left(\frac{\partial}{\partial u_{x}} V+i \frac{\partial}{\partial u_{y}} V\right)\right\rangle\right] \\
& +\frac{1}{k T(2 n+1)}\left[n(n-m+1)\left\langle X_{n+1 m} \frac{\partial}{\partial u_{z}} V\right\rangle-(n+1)(n+m)\left\langle X_{n-1 m} \frac{\partial}{\partial u_{z}} V\right\rangle\right], \quad(2.20)
\end{aligned}
$$


where the angular brackets designate the averages over $W$.

Now, let us suppose that a strong constant electric field $\mathbf{E}_{0}$ applied along the $z$ axis is suddenly switched on at time $t=0$. Our aim is to calculate the exact analytic solutions for the nonlinear response of relaxation processes customarily measured by experiment and arising from an assembly of noninteracting molecules. Equation (2.20) can be considerably simplified on noting that the vector $(\partial / \mu \partial \mathbf{u}) V$ has only a $z$ component, if the geometric axes of the molecule are chosen to be coincident with those of the molecular polarizability tensor, namely,

$$
\frac{\partial}{\mu \partial \mathbf{u}} V=-\left(E_{0}+\frac{\left(\alpha_{1}-\alpha_{2}\right)}{\mu} E_{0}^{2} u_{z}\right) \mathbf{k} .
$$

Using the recurrence relation between associated Legendre functions [19]

$$
(2 n+1) x P_{n}^{m}(x)=(n-m+1) P_{n+1}^{m}(x)+(n+m) P_{n-1}^{m}(x),
$$

and substituting Eq. (2.21) into Eq. (2.20), we obtain a fiveterm recurrence equation

$$
\begin{aligned}
& 2 \tau_{D} \frac{d}{d t}\left\langle X_{n m}\right\rangle+\left[n(n+1)-2 \sigma \frac{n(n+1)-3 m^{2}}{(2 n-1)(2 n+3)}\right]\left\langle X_{n m}\right\rangle \\
&=\xi\left[\frac{(n+1)(n+m)}{2 n+1}\left\langle X_{n-1 m}\right\rangle-\frac{n(n-m+1)}{2 n+1}\left\langle X_{n+1 m}\right\rangle\right] \\
& \quad+2 \sigma\left[\frac{(n+1)(n+m)(n+m-1)}{(2 n-1)(2 n+1)}\left\langle X_{n-2 m}\right\rangle\right. \\
&\left.-\frac{n(n-m+1)(n-m+2)}{(2 n+1)(2 n+3)}\left\langle X_{n+2 m}\right\rangle\right]
\end{aligned}
$$

where

$$
\xi=\frac{\mu E_{0}}{k T}, \quad \sigma=\frac{\left(\alpha_{1}-\alpha_{2}\right) E_{0}^{2}}{2 k T}
$$

The time-dependent quantities appropriate to dielectric and Kerr effect relaxation are the electric polarization

$$
P(t)=\mu N_{0}\left\langle P_{1}(\cos \vartheta)\right\rangle(t)=\mu N_{0}\left[\left\langle P_{1}(\cos \vartheta)\right\rangle(\infty)-f_{1}(t)\right]
$$

and the electric birefringence function

$$
\begin{aligned}
K(t) & =\frac{2 \pi N_{0}\left(\alpha_{1}^{o}-\alpha_{2}^{o}\right)}{\bar{n}}\left\langle P_{2}(\cos \vartheta)\right\rangle(t) \\
& =\frac{2 \pi N_{0}\left(\alpha_{1}^{o}-\alpha_{2}^{o}\right)}{\bar{n}}\left[\left\langle P_{2}(\cos \vartheta)\right\rangle(\infty)-f_{2}(t)\right],
\end{aligned}
$$

where $N_{0}$ is the concentration of molecules, $\alpha_{1}^{o}$ and $\alpha_{2}^{o}$ are the components of the optical polarizability due to the electric field of the light beam, $\bar{n}$ is the mean refractive index, and

$$
\begin{aligned}
f_{n}(t)= & \left\langle P_{n}(\cos \vartheta)\right\rangle(\infty)-\left\langle P_{n}(\cos \vartheta)\right\rangle(t) \\
& \times\left\langle P_{n}(\cos \vartheta)\right\rangle(\infty) \cdots
\end{aligned}
$$

is the relaxation function of order $n$. Moreover, another quantity which can be measured experimentally is the relaxation time $\tau_{n}$ defined as the area under the normalized relaxation function, namely,

$$
\tau_{n}=\int_{0}^{\infty} \frac{f_{n}(t)}{f_{n}(0)} d t
$$

Having switched on the constant electric field $\mathbf{E}_{0}$ the system will tend as $t \rightarrow \infty$ to a new equilibrium state with the Boltzmann distribution function

$$
W_{0}(\vartheta)=C \exp \left(-\frac{V}{k T}\right)=C \exp \left(\xi \cos \vartheta+\sigma \cos ^{2} \vartheta\right)
$$

where $C$ is the normalizing constant. The equilibrium averages of the spherical harmonics satisfy the recurrence relations (stationary state)

$$
\begin{aligned}
{[1-} & \left.2 \sigma \frac{1-3 m^{2} / n(n+1)}{(2 n-1)(2 n+3)}\right]\left\langle X_{n m}\right\rangle_{0} \\
= & \xi\left[\frac{(n+m)}{n(2 n+1)}\left\langle X_{n-1 m}\right\rangle_{0}-\frac{(n-m+1)}{(n+1)(2 n+1)}\left\langle X_{n+1 m}\right\rangle_{0}\right] \\
& +2 \sigma\left[\frac{(n+m)(n+m-1)}{n(2 n-1)(2 n+1)}\left\langle X_{n-2 m}\right\rangle_{0}\right. \\
& \left.-\frac{(n-m+1)(n-m+2)}{(n+1)(2 n+1)(2 n+3)}\left\langle X_{n+2 m}\right\rangle_{0}\right]
\end{aligned}
$$

where \langle\rangle$_{0}$ designates the equilibrium values averaged over the distribution function (2.28).

On setting $m=0$, we obtain from Eqs. (2.20) and (2.22) the hierarchy of differential-recurrence relations for the relaxation functions, namely,

$$
\begin{aligned}
& \frac{2 \tau_{D}}{n(n+1)} \frac{d}{d t} f_{n}(t)+\left[1-\frac{2 \sigma}{(2 n-1)(2 n+3)}\right] f_{n}(t) \\
& =\frac{\xi}{2 n+1}\left[f_{n-1}(t)-f_{n+1}(t)\right] \\
& \quad+2 \sigma\left[\frac{(n-1)}{(2 n-1)(2 n+1)} f_{n-2}(t)\right. \\
& \left.\quad-\frac{(n+2)}{(2 n+1)(2 n+3)} f_{n+2}(t)\right], \quad n=1,2, \ldots,
\end{aligned}
$$

where $f_{n}(t)$ is given by Eq. (2.26).

An equivalent system of equations has been derived in Refs. $[3,5,6]$ starting from the underlying Fokker-Planck equation. It should be noted that similar equations appear in the theory of dielectric relaxation of nematic liquid crystals $[7,11,20]$ and magnetic relaxation of single domain ferromagnetic particles [10,14,21-25]. In Refs. [7,10-14] we have developed an analytical method of evaluating the dynamic characteristics of the linear response of various physical systems governed by Eq. (2.30) and the particular cases 
$\xi=0$ and $\sigma=0$. Here we shall apply this approach to evaluate the corresponding nonlinear responses.

\section{EVALUATION OF THE RELAXATION TIME FOR $\xi=0$}

This is a nonlinear problem concerning the rise transient and relaxation time of the induced dipole Kerr effect, which was recently investigated in detail by Déjardin, Blaise, and Coffey [15]. However, below we reexamine this problem and present a solution which has the merit of being considerably simpler than that previously derived. Here the quantity of interest is the induced dipole relaxation time. Equation (2.30) can be considerably simplified and reduced to a threeterm recurrence relation as follows. We have

$$
\begin{aligned}
& \frac{2 \tau_{D}}{n(n+1)} \frac{d}{d t} f_{n}(t)+\left[1-2 \sigma \frac{1}{(2 n-1)(2 n+3)}\right] f_{n}(t) \\
& =2 \sigma\left[\frac{(n-1)}{(2 n-1)(2 n+1)} f_{n-2}(t)\right. \\
& \left.\quad-\frac{(n+2)}{(2 n+1)(2 n+3)} f_{n+2}(t)\right]
\end{aligned}
$$

with

$$
f_{0}(t)=0 \text {. }
$$

Since the reduced potential is of the form $\sigma \cos ^{2} \vartheta$, only the even Legendre polynomials will contribute to the initial conditions. These are

$$
\begin{aligned}
f_{2 n}(0) & =\left\langle P_{2 n}(\cos \vartheta)\right\rangle(\infty) \\
& =\frac{\int_{0}^{\pi} P_{2 n}(\cos \vartheta) e^{\sigma \cos ^{2} \vartheta} \sin \vartheta d \vartheta}{\int_{0}^{\pi} e^{\sigma \cos ^{2} \vartheta} \sin \vartheta d \vartheta} \\
& =\frac{\sigma^{n} \Gamma\left(n+\frac{1}{2}\right) M\left(n+\frac{1}{2}, 2 n+\frac{3}{2}, \sigma\right)}{2 \Gamma\left(2 n+\frac{3}{2}\right) M\left(\frac{1}{2}, \frac{3}{2}, \sigma\right)},
\end{aligned}
$$

$$
f_{2 n-1}(0)=0
$$

where $\Gamma(z)$ is the gamma function [19], $M(a, b, z)$ is the confluent hypergeometric (Kummer) function defined as [19]

$$
\begin{aligned}
M(a, b, z)= & 1+\frac{a}{b} \frac{z}{1 !}+\frac{a(a+1)}{b(b+1)} \frac{z^{2}}{2 !}+\frac{a(a+1)(a+2)}{b(b+1)(b+2)} \frac{z^{3}}{3 !} \\
& +\cdots
\end{aligned}
$$

Here $\left\langle P_{2 n}(\cos \vartheta)\right\rangle(0)=0$ because at time $t=0$ no field is present.

On applying the Laplace transform to Eq. (3.1) we obtain the algebraic equation

$$
\begin{gathered}
\frac{\widetilde{f}_{n}(s)}{\widetilde{f}_{n-2}(s)}\left[\frac{2 \tau_{D} s}{n(n+1)}+1-\frac{2 \sigma}{(2 n-1)(2 n+3)}\right. \\
\left.+\frac{2 \sigma(n+2)}{(2 n+1)(2 n+3)} \frac{\widetilde{f}_{n+2}(s)}{\widetilde{f}_{n}(s)}\right] \\
=\frac{2 \tau_{D}}{n(n+1)} \frac{f_{n}(0)}{\widetilde{f}_{n-2}(s)}+\frac{2 \sigma(n-1)}{4 n^{2}-1},
\end{gathered}
$$

where

$$
\widetilde{f}_{n}(s)=\int_{0}^{\infty} f_{n}(t) e^{-s t} d t .
$$

Following Refs. [7,10,11], we seek the solution of Eq. (3.6) in the form

$$
\widetilde{f}_{n}(s)=\widetilde{f}_{n-2} S_{n}(s)+q_{n}(s),
$$

where the continued fraction $S_{n}(s)$, defined as

$$
S_{n}(s)=\frac{\frac{2 \sigma(n-1)}{4 n^{2}-1}}{\frac{2 \tau_{D} s}{n(n+1)}+1-\frac{2 \sigma}{(2 n-1)(2 n+3)}+\frac{2 \sigma(n+2)}{(2 n+1)(2 n+3)} S_{n+2}(s)},
$$

is the solution of the homogeneous Eq. (3.6) [with $f_{n}(0)=0$ ].

As demonstrated in Ref. [6], the solution of the homogeneous equation (3.6) allows one to evaluate the Laplace transform of $\left\langle P_{2}(\cos \vartheta)\right\rangle(t)$, which is

$$
\int_{0}^{\infty}\left\langle P_{2}(\cos \vartheta)\right\rangle(t) e^{-s t} d t=\frac{S_{2}(s)}{s} .
$$

However in order to obtain the Kerr effect relaxation time from Eq. (2.27) we must obtain a solution of the inhomogeneous Eq. (2.30).
On substituting Eq. (3.8) into Eq. (3.6) and using Eq. (3.9), we have

$$
q_{n}=\left[\frac{\tau_{D}}{\sigma} a_{n} f_{n}(0)-b_{n} q_{n+2}\right] S_{n}(s),
$$

where

$$
a_{n}=\frac{4 n^{2}-1}{n\left(n^{2}-1\right)}, \quad b_{n}=\frac{(n+2)(2 n-1)}{(n-1)(2 n+3)} .
$$


Thus,

$$
\widetilde{f}_{n}(s)=\left\{\widetilde{f}_{n-2}(s)+\frac{\tau_{D}}{\sigma} a_{n} f_{n}(0)-b_{n} q_{n+2}\right\} S_{n}(s) .
$$

Since all the relaxation functions with odd indices are equal to zero [see Eq. (3.4)], we can solve Eq. (3.1) for even $n$ only. In particular for $n=2$ we have

$$
\widetilde{f}_{2}(s)=\left\{\frac{5 \tau_{D}}{2 \sigma} f_{2}(0)-\frac{12}{7} q_{4}\right\} S_{2}(s) .
$$

We obtain by iterating Eq. (3.10) for $n=4,6$ and so on in Eq. (3.13),

$$
\begin{aligned}
\widetilde{f}_{2}(s)= & \frac{3 \tau_{D}}{2 \sigma} \sum_{n=1}^{\infty}(-1)^{n+1} f_{2 n}(0) a_{2 n} \prod_{k=1}^{n} b_{2 k-2} S_{2 k}(s) \\
= & \frac{3 \sqrt{\pi} \tau_{D}}{8 \sigma} \sum_{n=1}^{\infty}(-1)^{n+1} \frac{(4 n+1) \Gamma(n)}{\Gamma(n+3 / 2)} f_{2 n}(0) \\
& \times \prod_{k=1}^{n} S_{2 k}(s) .
\end{aligned}
$$

The Kerr effect relaxation time $\tau_{2}$, defined from Eq. (2.27) for $n=2$ is

$$
\tau_{2}=\frac{\int_{0}^{\infty} f_{2}(t) d t}{f_{2}(0)}=\frac{\tilde{f}_{2}(0)}{f_{2}(0)}
$$

so that from Eq. (3.14) we have

$$
\begin{aligned}
\tau_{2}= & \frac{3 \sqrt{\pi} \tau_{D}}{8 \sigma f_{2}(0)} \\
& \times \sum_{n=1}^{\infty}(-1)^{n+1} \frac{(4 n+1) \Gamma(n)}{\Gamma(n+3 / 2)} f_{2 n}(0) \prod_{k=1}^{n} S_{2 k}(0),
\end{aligned}
$$

where $\widetilde{S_{n}}(0)$ is given by Eq. (3.9) at $s=0$.

Noting that the equilibrium averages $\left\langle P_{n}(\cos \vartheta)\right\rangle(\infty)$ satisfy Eq. (2.29) for $m=0$ with $\xi=0$, we have

$$
S_{n}(0)=\frac{\left\langle P_{n}(\cos \vartheta)\right\rangle(\infty)}{\left\langle P_{n-2}(\cos \vartheta)\right\rangle(\infty)} \text {. }
$$

Taking into account Eq. (3.17), the initial conditions $f_{2 n}(0)$ are

$f_{2 n}(0)=S_{2 n}(0) S_{2 n-2}(0) \ldots S_{2}(0)=\prod_{k=1}^{n} S_{2 k}(0)$.

Thus on using Eqs. (3.3) and (3.17), we obtain from Eq. (3.15) the exact analytical solution for the relaxation time, namely,

$$
\tau_{2}=\frac{45 \pi \tau_{D}}{64 M(1 / 2,3 / 2, \sigma) M(3 / 2,7 / 2, \sigma)} \sum_{n=1}^{\infty}\left(-\frac{\sigma^{2}}{4}\right)^{n-1}
$$

$$
\times \frac{(4 n+1) \Gamma(2 n) M^{2}(n+1 / 2,2 n+3 / 2, \sigma)}{(2 n+1) \Gamma^{2}(2 n+3 / 2)}
$$

We remark that Eq. (35) of Ref. [15] differs in form from Eq. (3.18), however, it can be reduced to Eq. (3.18). We also remark that all the confluent hypergeometric functions appearing in Eq. (3.18) may be expressed in terms of the more familiar error function of imaginary argument, viz.,

$$
\operatorname{erf} i(x)=\frac{2}{\sqrt{\pi}} \int_{0}^{x} e^{t^{2}} d t
$$

In particular ([26], pp. 580 and 581)

$$
\begin{gathered}
M\left(\frac{1}{2}, \frac{3}{2}, z\right)=\frac{1}{2}\left(\frac{\pi}{z}\right)^{1 / 2} \operatorname{erf} i(\sqrt{z}), \\
M\left(\frac{3}{2}, \frac{7}{2}, z\right)=\frac{15}{8 z^{2}}\left[3 e^{z}-\frac{3+2 z}{2}\left(\frac{\pi}{z}\right)^{1 / 2} \operatorname{erf} i(\sqrt{z})\right] .
\end{gathered}
$$

Equations for the other $M$ functions occurring in Eq. (3.18) may be obtained from Table 7.11.2 of Ref. [26] and the recurrence relations for the confluent hypergeometric function.

In the limit $\sigma \rightarrow 0$, on using the Taylor expansion (3.5), we obtain

$$
\frac{\tau_{2}}{\tau_{D}} \approx \frac{1}{3}+\frac{2}{63} \sigma-\frac{212}{33075} \sigma^{2}+O\left(\sigma^{3}\right) .
$$

In the opposite limits $\sigma \rightarrow \pm \infty$, on using the asymptotic expansion of the confluent hypergeometric function [19]

$$
\begin{array}{r}
M(a, b, z) \sim \frac{e^{i \pi a} z^{-\alpha} \Gamma(b)}{\Gamma(b-a)}\left[1+O\left(\frac{1}{-z}\right)\right] \\
+\frac{e^{z} z^{a-b} \Gamma(b)}{\Gamma(a)}\left[1+O\left(\frac{1}{z}\right)\right],
\end{array}
$$

we have

$$
\begin{aligned}
\frac{\tau_{2}}{\tau_{D}} & \sim \frac{3 \sqrt{\pi}}{8 \sigma}\left[4 \sum_{n=0}^{\infty}(-1)^{n} \frac{\Gamma(n+2)}{\Gamma\left(n+\frac{5}{2}\right)}+\sum_{n=0}^{\infty}(-1)^{n} \frac{\Gamma(n+1)}{\Gamma\left(n+\frac{5}{2}\right)}\right] \\
& =\frac{1}{2 \sigma}\left[4{ }_{2} F_{1}\left(1,2 ; \frac{5}{2} ;-1\right)+{ }_{2} F_{1}\left(1,1 ; \frac{5}{2} ;-1\right)\right] \\
& =\frac{3}{2 \sigma}, \quad \text { for } \sigma>0
\end{aligned}
$$

and

$$
\begin{aligned}
\frac{\tau_{2}}{\tau_{D}} \sim & \frac{3}{\sqrt{\pi}|\sigma|}\left[\sum_{n=0}^{\infty}(-1)^{n} \frac{\Gamma\left(n+\frac{3}{2}\right)}{(2 n+3) \Gamma(n+2)}\right. \\
& \left.+\sum_{n=0}^{\infty}(-1)^{n} \frac{\Gamma\left(n+\frac{3}{2}\right)}{(2 n+2) \Gamma(n+2)}\right] \\
= & \frac{3}{4|\sigma|}\left[\frac{2}{3}{ }_{3} F_{2}\left(1, \frac{3}{2}, \frac{3}{2} ; 2, \frac{5}{2} ;-1\right)+{ }_{3} F_{2}\left(1,1, \frac{3}{2} ; 2,2 ;-1\right)\right] \\
= & \frac{3(1-\ln 2)}{|\sigma|} \quad \text { for } \sigma<0 .
\end{aligned}
$$




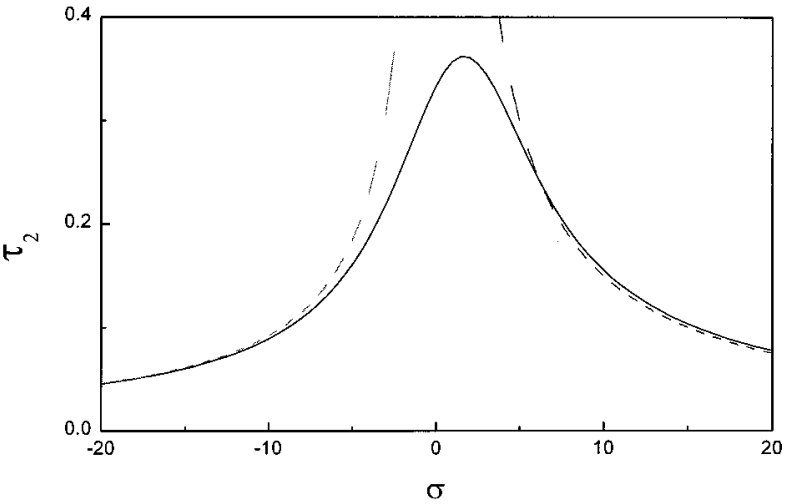

FIG. 1. Relaxation time $\tau_{2}$ as a function of $\sigma$ (solid line) at $\tau_{D}=1$. The dashed lines are the asymptotic dependences given by Eqs. (3.21) and (3.22).

Here we have used the following equations for the hypergeometric functions ${ }_{q} F_{p}\left(a_{1}, \ldots a_{q} ; b_{1}, \ldots b_{p} ; z\right)[26]$ :

$$
\begin{gathered}
{ }_{2} F_{1}\left(1,1 ; \frac{5}{2} ; z\right)=\frac{3}{z}\left[1-\left(\frac{1-z}{z}\right)^{1 / 2} \arcsin \sqrt{z}\right], \\
{ }_{2} F_{1}\left(1,2 ; \frac{5}{2} ; z\right)=\frac{3}{2 z}\left[\frac{\arcsin \sqrt{z}}{\sqrt{z(1-z)}}-1\right], \\
{ }_{3} F_{2}\left(1,1, \frac{3}{2} ; 2,2 ; z\right)=\frac{4}{z} \ln \frac{2(1-\sqrt{1-z})}{z}, \\
{ }_{3} F_{2}\left(1, \frac{3}{2}, \frac{3}{2} ; 2, \frac{5}{2} ; z\right)=\frac{6}{z}\left[\frac{\arcsin \sqrt{z}}{\sqrt{z}}-1\right] .
\end{gathered}
$$

The behavior of the relaxation time $\tau_{2}$ as a function of $\sigma$ is shown in Fig. 1 for $\sigma$ values in the range $-20<\sigma<20$. For positive $\sigma$ values this positive Kerr-effect relaxation time passes through a maximum at a certain value of $\sigma$ before decreasing monotonically to zero with increasing $\sigma$ as a result already obtained by Watanabe and Morita [6] and Déjardin, Blaise, and Coffey [15]. For negative $\sigma$ values, the $\tau_{2}$ decays monotonically to zero with increasing $|\sigma|$. Moreover, one can see that the asymptotic Eqs. (3.21) and (3.22) closely fit the exact solution for $\sigma>6$ and $\sigma<-10$. The surprising increase in $\tau_{2}$ at intermediate electric fields was also predicted from a numerical solution of Eq. (3.1) and observed experimentally by Tolles [27].

\section{EVALUATION OF THE RELAXATION TIMES FOR $\sigma=0$}

If the contribution of the induced dipole moment is negligible in comparison with that of the permanent dipole moment we have, on putting $\sigma=0$ in Eq. (2.30),

$$
\begin{aligned}
2 \tau_{D} & \frac{d}{d t} f_{n}(t)+n(n+1) f_{n}(t) \\
& =\frac{\xi n(n+1)}{(2 n+1)}\left[f_{n-1}(t)-f_{n+1}(t)\right] .
\end{aligned}
$$

The initial conditions are therefore given by

$f_{n}(0)=\int_{0}^{\pi} P_{n}(\cos \vartheta) W_{0}(\vartheta) \sin \vartheta d \vartheta=\frac{I_{n+1 / 2}(\xi)}{I_{1 / 2}(\xi)}$,

where $W_{0}(\vartheta)$ is the equilibrium Boltzmann distribution function

$$
W_{0}(\boldsymbol{\vartheta})=C \exp \left(\frac{\left(\boldsymbol{\mu} \cdot \mathbf{E}_{0}\right)}{k T}\right)=\frac{\xi}{4 \pi \operatorname{sh}(\xi)} \exp (\xi \cos \vartheta)
$$

and $I_{\nu}(z)$ is the modified Bessel function of the first kind [19].

On applying the Laplace transform to Eq. (4.1), we obtain

$$
\begin{aligned}
{\left[2 \tau_{D} s+n(n+1)\right] \widetilde{f}_{n}(s)=} & \frac{\xi n(n+1)}{(2 n+1)}\left[\widetilde{f}_{n-1}(s)-\widetilde{f}_{n+1}(s)\right] \\
& +2 \tau_{D} f_{n}(0)
\end{aligned}
$$

The exact analytic solution of Eq. (4.4) is in like manner (see also [7,12]):

$$
\begin{aligned}
\widetilde{f}_{n}(s)= & \widetilde{f}_{n-1}(s) S_{n}(s)+\frac{2 \tau_{D}}{\xi} \sum_{k=1}^{\infty}(-1)^{k+1} \\
& \times \frac{(2 n+2 k-1)}{(n+k-1)(n+k)} f_{n+k-1}(0) \prod_{m=n}^{n+k-1} S_{m}(s)
\end{aligned}
$$

In particular for $n=1$ and $n=2$ we have

$$
\widetilde{f}_{1}(s)=\frac{2 \tau_{D}}{\xi} \sum_{n=1}^{\infty}(-1)^{n+1} \frac{(2 n+1)}{n(n+1)} f_{n}(0) \prod_{k=1}^{n} S_{k}(s),
$$

$$
\begin{aligned}
\widetilde{f}_{2}(s)= & \widetilde{f}_{1}(s) S_{2}(s)+\frac{2 \tau_{D}}{\xi} \sum_{n=2}^{\infty}(-1)^{n} \frac{(2 n+1)}{n(n+1)} \\
& \times f_{n}(0) \prod_{k=2}^{n} S_{k}(s) \\
= & \frac{3 \tau_{D}}{\xi} f_{1}(0) S_{1}(s) S_{2}(s)+\frac{2 \tau_{D}}{\xi}\left[1-S_{1}(s) S_{2}(s)\right] \\
& \times \sum_{n=2}^{\infty}(-1)^{n} \frac{(2 n+1)}{n(n+1)} f_{n}(0) \sum_{k=2}^{n} S_{k}(s),
\end{aligned}
$$

where the continued fraction $S_{n}(s)$ is given by

$$
S_{n}(s)=\frac{\xi}{\frac{2 \tau_{D} s(2 n+1)}{n(n+1)}+2 n+1+\xi S_{n+1}(s)} .
$$


The present problem has also been considered by Watanabe and Morita [6]. However, they did not give exact expressions for the relaxation times, nor did they solve the three-term recurrence relations for the polarization and rise transient in continued fraction form. As shown in Ref. [6], the solution of the homogeneous equation (4.4) allows one to evaluate the Laplace transform of $\left\langle P_{1}(\cos \vartheta)\right\rangle(t)$ and $\left\langle P_{2}(\cos \vartheta)\right\rangle(t)$, which is in our notation

$$
\begin{gathered}
\int_{0}^{\infty}\left\langle P_{1}(\cos \vartheta)\right\rangle(t) e^{-s t} d t=\frac{S_{1}(s)}{s}, \\
\int_{0}^{\infty}\left\langle P_{2}(\cos \vartheta)\right\rangle(t) e^{-s t} d t=\frac{1}{s}\left[1-\frac{3}{\xi}\left(1+s \tau_{D}\right) S_{1}(s)\right] .
\end{gathered}
$$

Using the definition of the relaxation time, namely,

$$
\tau_{n}=\frac{\widetilde{f}_{n}(0)}{f_{n}(0)} \quad(n=1,2)
$$

we have from Eqs. (4.6) and (4.7)

$$
\begin{gathered}
\tau_{1}=\frac{2 \tau_{D}}{\xi} \sum_{n=1}^{\infty}(-1)^{n+1} \frac{(2 n+1) f_{n}(0)}{n(n+1) f_{1}(0)} \prod_{k=1}^{n} S_{n}(0), \\
\tau_{2}=\tau_{1}+\frac{2 \tau_{D}}{\xi} \sum_{n=2}^{\infty}(-1)^{n} \frac{(2 n+1) f_{n}(0)}{n(n+1) f_{2}(0)} \prod_{k=2}^{n} S_{k}(0),
\end{gathered}
$$

where

$$
S_{n}(0)=\frac{\xi}{2 n+1+\xi S_{n+1}(0)}=\frac{I_{n+1 / 2}(\xi)}{I_{n-1 / 2}(\xi)}
$$

since the modified Bessel functions $I_{\nu}(z)$ satisfy the recurrence relation [19]

$$
I_{\nu-1}(z)-I_{\nu+1}(z)=\frac{2 \nu}{z} I_{\nu}(z)
$$

which can be represented as the continued fraction

$$
\frac{I_{\nu}(z)}{I_{\nu-1}(z)}=\frac{z}{2 \nu+z \frac{I_{\nu+1}(z)}{I_{\nu}(z)}}
$$

We remark that the modified Bessel functions of the second kind $K_{\nu}(z)$ will also satisfy this recurrence relation. However, they must be discarded as a solution of Eq. (4.8) as they are infinite at $z=0$.

On substituting Eq. (4.14) into Eqs. (4.12) and (4.13), we obtain

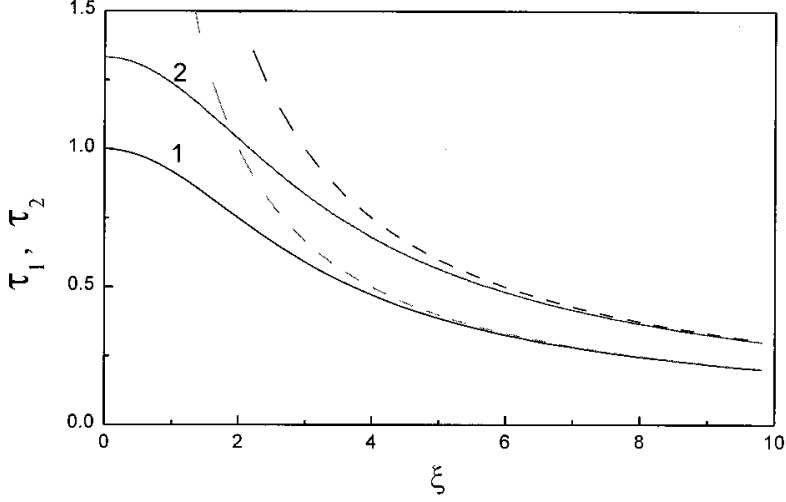

FIG. 2. Relaxation times $\tau_{1}$ (curve 1) and $\tau_{2}$ (curve 2) as a function of $\xi$ at $\tau_{D}=1$. The dashed lines are the asymptotic dependences given by Eqs. (4.19) and (4.20).

$$
\tau_{1}=\frac{2 \tau_{D}}{\xi I_{1 / 2}(\xi) I_{3 / 2}(\xi)} \sum_{n=1}^{\infty}(-1)^{n+1} \frac{(2 n+1)}{n(n+1)} I_{n+1 / 2}^{2}(\xi),
$$

$$
\tau_{2}=\tau_{1}+\frac{2 \tau_{D}}{\xi I_{3 / 2}(\xi) I_{5 / 2}(\xi)} \sum_{n=2}^{\infty}(-1)^{n} \frac{(2 n+1)}{n(n+1)} I_{n+1 / 2}^{2}(\xi) .
$$

In the limit $\xi \rightarrow 0$ on using the Taylor expansion [19]

$$
I_{\nu}(z)=\left(\frac{z}{2}\right)^{\nu} \sum_{k=0}^{\infty} \frac{(z / 2)^{2 k}}{k ! \Gamma(k+\nu+1)},
$$

we have

$$
\frac{\tau_{1}}{\tau_{D}} \approx 1-\frac{4}{45} \xi^{2}+\frac{89}{9450} \xi^{4}+O\left(\xi^{6}\right),
$$

$$
\frac{\tau_{2}}{\tau_{D}} \approx \frac{4}{3}-\frac{13}{126} \xi^{2}+\frac{673}{66150} \xi^{4}+O\left(\xi^{6}\right) .
$$

In the opposite limit $\xi \rightarrow \infty$, on using the asymptotic expansion

$$
I_{\nu}(z) \sim \frac{e^{z}}{\sqrt{2 z \pi}}\left[1+O\left(\frac{1}{z}\right)\right],
$$

we find

$$
\begin{aligned}
& \frac{\tau_{1}}{\tau_{D}} \sim \frac{2}{\xi}, \\
& \frac{\tau_{2}}{\tau_{D}} \sim \frac{3}{\xi} .
\end{aligned}
$$

The behavior of the relaxation times $\tau_{1}$ and $\tau_{2}$ as functions of $\xi$ is shown in Fig. 2. Both $\tau_{1}$ and $\tau_{2}$ decrease monotonically to zero with increasing $\xi$. One can see in this figure that the asymptotic Eqs. (4.19) and (4.20) closely fit the exact solution for $\xi>5$. 


\section{EVALUATION OF THE RELAXATION FUNCTIONS AND RELAXATION TIMES IN THE GENERAL CASE}

Equation (2.30) can be transformed into the matrix three-term differential-recurrence equation

$$
\tau_{D} \frac{d}{d t} \mathbf{C}_{n}(t)=\mathbf{Q}_{n}^{-} \mathbf{C}_{n-1}(t)+\mathbf{Q}_{n} \mathbf{C}_{n}(t)+\mathbf{Q}_{n}^{+} \mathbf{C}_{n+1}(t)
$$

if we arrange it as follows $[7,14]$ :

$$
\begin{aligned}
\tau_{D} \frac{d}{d t}\left(\begin{array}{c}
f_{2 n-1}(t) \\
f_{2 n}(t)
\end{array}\right)= & \left(\begin{array}{cc}
\frac{4 \sigma n(n-1)(2 n-1)}{(4 n-1)(4 n-3)} & \frac{\xi n(2 n-1)}{(4 n-1)} \\
0 & \frac{2 \sigma n(2 n-1)(2 n+1)}{(4 n-1)(4 n+1)}
\end{array}\right)\left(\begin{array}{l}
f_{2 n-3}(t) \\
f_{2 n-2}(t)
\end{array}\right) \\
& +\left(\begin{array}{c}
n(2 n-1)\left[\frac{2 \sigma}{(4 n-3)(4 n+1)}-1\right.
\end{array}\right]-\frac{\xi n(2 n-1)}{(4 n-1)} \\
\frac{\xi n(2 n+1)}{(4 n+1)} & \left.n(2 n+1)\left[\begin{array}{c}
\frac{2 \sigma}{(4 n-1)(4 n+3)}-1
\end{array}\right]\right)\left(\begin{array}{c}
f_{2 n-1}(t) \\
f_{2 n}(t)
\end{array}\right) \\
& +\left(\begin{array}{cc}
-\frac{2 \sigma n(2 n-1)(2 n+1)}{(4 n-1)(4 n+1)} & 0 \\
-\frac{\xi n(2 n+1)}{(4 n+1)} & -\frac{4 \sigma n(n+1)(2 n+1)}{(4 n+1)(4 n+3)}
\end{array}\right)\left(\begin{array}{l}
f_{2 n+1}(t) \\
f_{2 n+2}(t)
\end{array}\right) .
\end{aligned}
$$

On applying the general method of solution of the matrix three-term recurrence equation $(5.1)$, suggested in Refs. [7, 13]], we obtain a solution for the Laplace transform $\widetilde{\mathbf{C}}_{1}(s)$ in terms of matrix continued fractions

$$
\left(\begin{array}{c}
\tilde{f}_{1}(s) \\
\widetilde{f}_{2}(s)
\end{array}\right)=\tau_{D}\left[\tau_{D} s \mathbf{I}-\mathbf{Q}_{1}-\mathbf{Q}_{1}^{+} \mathbf{S}_{2}(s)\right]^{-1}\left\{\mathbf{C}_{1}(0)+\sum_{n=2}^{\infty} \prod_{k=2}^{n} \mathbf{Q}_{k-1}^{+} \mathbf{S}_{k}(s)\left(\mathbf{Q}_{k}^{-}\right)^{-1} \mathbf{C}_{n}(0)\right\},
$$

where $\mathbf{I}$ is the $2 \times 2$ identity matrix, $\mathbf{Q}_{n}, \mathbf{Q}_{n}^{ \pm}$are the $2 \times 2$ matrices given in Eq. (5.2), the matrix continued fraction $\mathbf{S}_{n}(s)$ is defined as

$$
\mathbf{S}_{n}(s)=\left[\tau_{D} s \mathbf{I}-\mathbf{Q}_{n}-\mathbf{Q}_{n}^{+} \mathbf{S}_{n+1}(s)\right]^{-1} \mathbf{Q}_{n}^{-}
$$

The initial value vectors

$$
\mathbf{C}_{n}(0)=\left(\begin{array}{c}
f_{2 n-1}(0) \\
f_{2 n}(0)
\end{array}\right)=\left(\begin{array}{c}
\left\langle P_{2 n-1}(\cos \vartheta)\right\rangle_{0} \\
\left\langle P_{2 n}(\cos \vartheta)\right\rangle_{0}
\end{array}\right)
$$

may be evaluated from the recurrence relation

$$
\left[1-\frac{2 \sigma}{(2 n-1)(2 n+3)}\right]\left\langle P_{n}\right\rangle_{0}=\frac{\xi}{2 n+1}\left[\left\langle P_{n-1}\right\rangle_{0}-\left\langle P_{n+1}\right\rangle_{0}\right]+\left[\frac{2 \sigma(n-1)}{(2 n-1)(2 n+1)}\left\langle P_{n-2}\right\rangle_{0}-\frac{2 \sigma(n+2)}{(2 n+1)(2 n+3)}\left\langle P_{n+2}\right\rangle_{0}\right],
$$

where the three first members of the hierarchy are $[7,13]$

$$
\begin{gathered}
\left\langle P_{0}\right\rangle_{0}=1, \\
\left\langle P_{1}\right\rangle_{0}=\frac{1}{\sqrt{\sigma}\left[(\operatorname{coth} \xi+1) D\left(\sqrt{\sigma}+\frac{\xi}{2 \sqrt{\sigma}}\right)+(\operatorname{coth} \xi-1) D\left(\sqrt{\sigma}-\frac{\xi}{2 \sqrt{\sigma}}\right)\right]}-\frac{\xi}{2 \sigma}, \\
\left\langle P_{2}\right\rangle_{0}=\frac{\frac{3}{2}\left(\operatorname{coth} \xi-\frac{\xi}{2 \sigma}\right)}{\sqrt{\sigma}\left[(\operatorname{coth} \xi+1) D\left(\sqrt{\sigma}+\frac{\xi}{2 \sqrt{\sigma}}\right)+(\operatorname{coth} \xi-1) D\left(\sqrt{\sigma}-\frac{\xi}{2 \sqrt{\sigma}}\right)\right]}+\frac{3 \xi^{2}}{8 \sigma^{2}}-\frac{3}{4 \sigma}-\frac{1}{2} .
\end{gathered}
$$




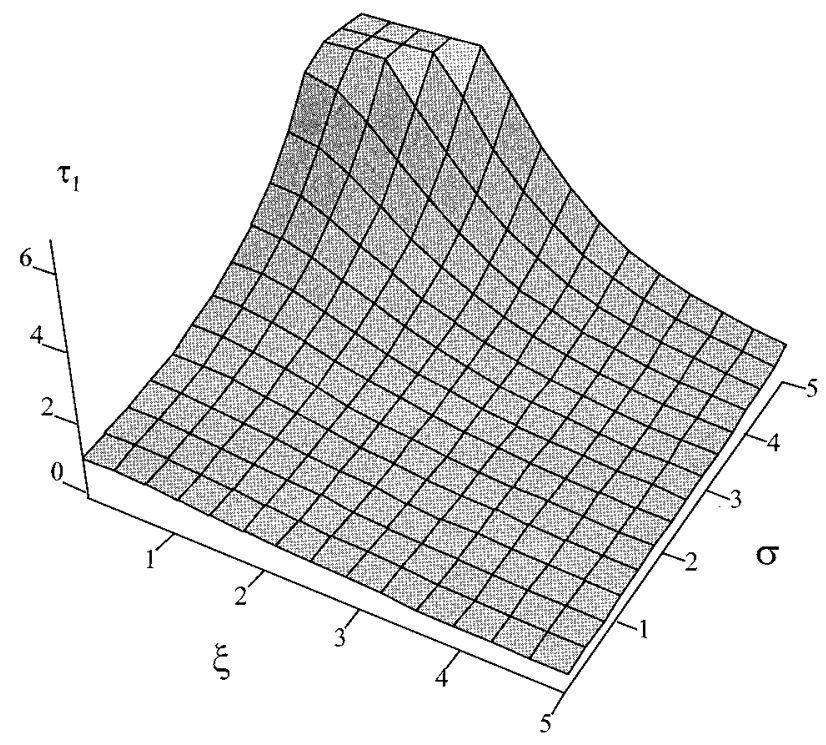

FIG. 3. Relaxation time $\tau_{1}$ as a function of $\xi$ and $\sigma$ at $0.01<\xi<5$ and $0.01<\sigma<5$ and at $\tau_{D}=1$.

Here

$$
D(x)=\frac{\sqrt{\pi}}{2} e^{-x^{2}} \operatorname{erfi}(x)=e^{-x^{2}} \int_{0}^{x} e^{t^{2}} d t .
$$

Equation (5.6) follows from Eq. (2.29) at $m=0$. It should be noted however that this upward iteration is unstable and one should use it with caution.

However the initial value vectors $\mathbf{C}_{n}(0)$ can be evaluated more efficiently with the aid of matrix continued fractions. Thus, let us transform Eq. (5.6) to the matrix form

$$
\begin{gathered}
\mathbf{Q}_{n}^{-}\left(\begin{array}{c}
\left\langle P_{2 n-3}(\cos \vartheta)\right\rangle_{0} \\
\left\langle P_{2 n-2}(\cos \vartheta)\right\rangle_{0}
\end{array}\right)+\mathbf{Q}_{n}\left(\begin{array}{c}
\left\langle P_{2 n-1}(\cos \vartheta)\right\rangle_{0} \\
\left\langle P_{2 n}(\cos \vartheta)\right\rangle_{0}
\end{array}\right) \\
+\mathbf{Q}_{n}^{+}\left(\begin{array}{c}
\left\langle P_{2 n+1}(\cos \vartheta)\right\rangle_{0} \\
\left\langle P_{2 n+2}(\cos \vartheta)\right\rangle_{0}
\end{array}\right)=0
\end{gathered}
$$

or

$$
\mathbf{Q}_{n}^{-} \mathbf{C}_{n-1}(0)+\mathbf{Q}_{n} \mathbf{C}_{n}(0)+\mathbf{Q}_{n}^{+} \mathbf{C}_{n+1}(0)=0 .
$$

The solution of Eq. (5.7) is then given by

$$
\begin{aligned}
\mathbf{C}_{n}(0) & =\left[-\mathbf{Q}_{n}-\mathbf{Q}_{n}^{+} \mathbf{S}_{n+1}(0)\right]^{-1} \mathbf{Q}_{n}^{-} \mathbf{C}_{n-1}(0) \\
& =\mathbf{S}_{n}(0) \mathbf{C}_{n-1}(0) \\
& =\mathbf{S}_{n}(0) \mathbf{S}_{n-1}(0) \ldots \mathbf{S}_{1}(0)\left(\begin{array}{l}
0 \\
1
\end{array}\right) \quad(n=1,2 \ldots) .
\end{aligned}
$$

In particular, for $n=1$ we have

$$
\mathbf{C}_{1}(0)=\left(\begin{array}{l}
f_{1}(0) \\
f_{2}(0)
\end{array}\right)=\left(\begin{array}{l}
\left\langle P_{1}(\cos \vartheta)\right\rangle_{0} \\
\left\langle P_{2}(\cos \vartheta)\right\rangle_{0}
\end{array}\right)=\mathbf{S}_{1}(0)\left(\begin{array}{l}
0 \\
1
\end{array}\right)
$$

We can now evaluate the relaxation times $\tau_{1}$ and $\tau_{2}$ from Eqs. (5.3) and (5.9) (see Figs. 3 and 4). We remark that the solution in the form of Eq. (5.3) is mainly needed for the

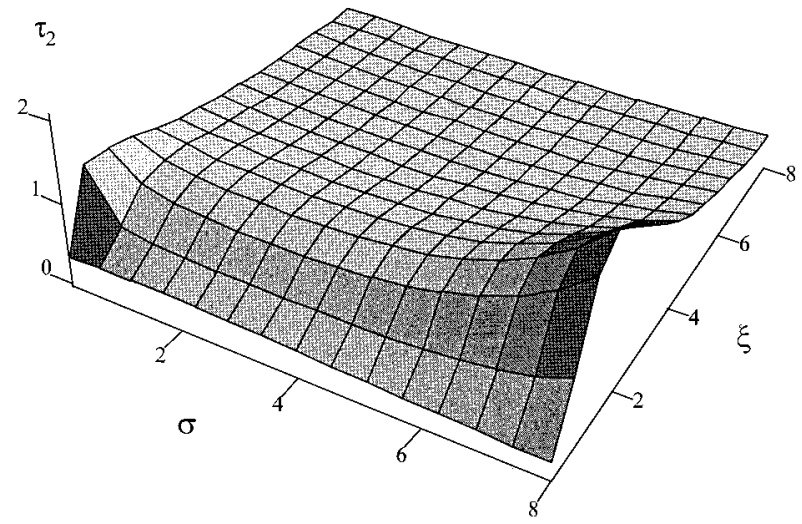

FIG. 4. Relaxation time $\tau_{2}$ as a function of $\xi$ and $\sigma$ at $0.01<\xi<8$ and $0.01<\sigma<8$ and at $\tau_{D}=1$.

calculation of the relaxation times. The Laplace transforms of $\left\langle P_{1}(\cos \vartheta)\right\rangle(t)$ and $\left\langle P_{2}(\cos \vartheta)\right\rangle(t)$ have a simpler representation as

$\left(\begin{array}{l}\int_{0}^{\infty}\left\langle P_{1}(\cos \vartheta)\right\rangle(t) e^{-s t} d t \\ \int_{0}^{\infty}\left\langle P_{2}(\cos \vartheta)\right\rangle(t) e^{-s t} d t\end{array}\right)=s^{-1} \mathbf{S}_{1}(s)\left(\begin{array}{l}0 \\ 1\end{array}\right)$.

On taking into account Eqs. (2.26), (5.10), and (5.9), we can simplify Eq. (5.3) as follows:

$$
\left(\begin{array}{c}
\tilde{f}_{1}(s) \\
\widetilde{f}_{2}(s)
\end{array}\right)=s^{-1}\left[\mathbf{S}_{1}(0)-\mathbf{S}_{1}(s)\right]\left(\begin{array}{l}
0 \\
1
\end{array}\right) .
$$

Thus, in order to calculate the nonlinear dielectric and dynamic Kerr effect step-on responses we simply need to evaluate the matrix continued fraction $\mathbf{S}_{1}(s)$.

\section{RESULTS AND DISCUSSION}

The behavior of the real and imaginary parts of the onesided Fourier transforms of the normalized relaxation functions, defined as

$$
\chi_{n}(\omega)=\frac{\widetilde{f_{n}}(i \omega)}{\tau_{D} f_{n}(0)},
$$

is shown in Figs. 5-8. Here the spectra evaluated from the exact solutions given by Eqs. (3.14), (4.7), and (4.8), are compared with the Lorentz spectrum

$$
\chi_{D n}(\omega)=\frac{\tau_{n} / \tau_{D}}{1+i \omega \tau_{n}}
$$

where $\tau_{n}$ are the relaxation times calculated from Eqs. (3.18), (4.1), and (4.15). Equation (6.2) corresponds to the representation of the relaxation functions $f_{n}(t)$ by a purely exponential term

$$
f_{n}(t)=f_{n}(0) e^{-t / \tau_{n}}
$$

It is apparent from Figs. 5-8 that Lorentzian behavior is obtained for the spectra $\chi_{1}(\omega)$ for arbitrary $\xi$ and at $\sigma \approx 0$ and 


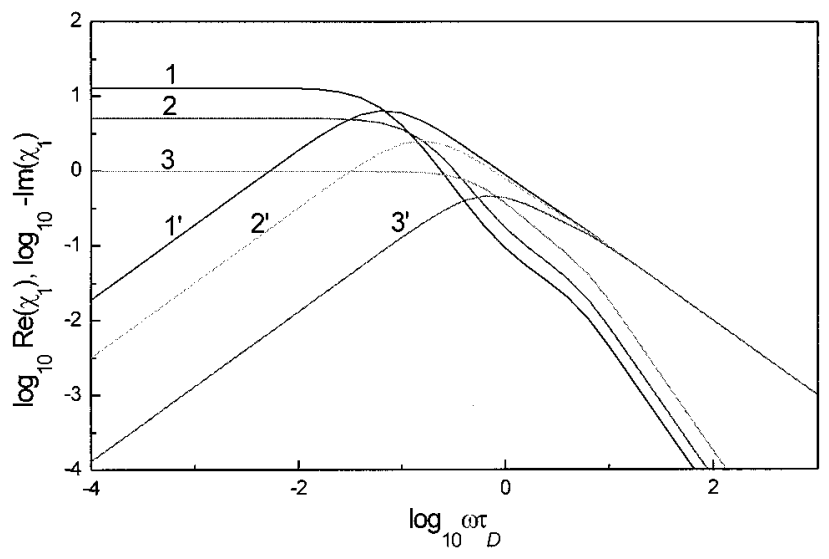

FIG. 5. Real (curves 1,2,3) and imaginary (curves $1^{\prime}, 2^{\prime}, 3^{\prime}$ ) parts of the spectrum $\chi_{1}(\omega)$ of the normalized relaxation function at $\sigma=5$. Curves $1,1^{\prime}, 2,2^{\prime}$, and $3,3^{\prime}$ correspond to $\xi=0.01$, 1 , and 5 , respectively.

$\chi_{2}(\omega)$ for arbitrary $\sigma$ and $\xi=0$ (cf. Ref. [15]). Thus, in these cases alone the relaxation functions $f_{1}(t)$ and $f_{2}(t)$ can be approximated by a single exponential. In all other cases the decay of the relaxation functions $f_{n}(t)$ has a more complicated behavior. This may be explained as follows. The relaxation dynamics in the potential given by Eq. (2.5) (which has in general two potential wells) is determined by two relaxation processes. One relaxation (activation) process governs the crossing of the potential barrier between positions of equilibrium by a current of molecules. Another process describes orientational relaxation inside the wells. In the case of nonpolarizable molecules, when the potential (2.5) transforms to a single well, we observe one relaxation process only. As is apparent from Figs. 3 and 4 the permanent dipole contribution suppresses the activation process. A similar result has been obtained for the linear response in magnetic relaxation of single domain ferromagnetic particles with high anisotropy barriers in the presence of a strong constant magnetic field following an infinitesimal change in that field [14].

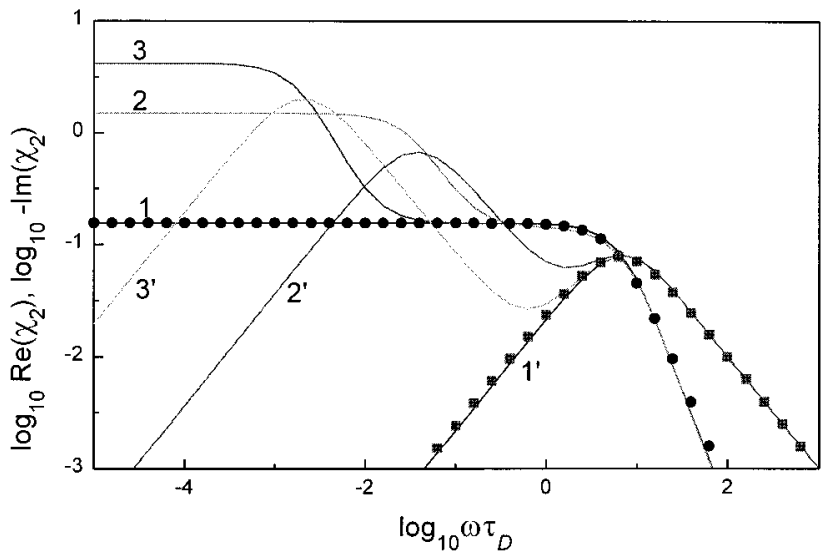

FIG. 6. Real (curves 1,2,3) and imaginary (curves $1^{\prime}, 2^{\prime}, 3^{\prime}$ ) parts of the spectrum $\chi_{2}(\omega)$ of the normalized relaxation function at $\sigma=10$. Curves $1,1^{\prime}, 2,2^{\prime}$, and $3,3^{\prime}$ correspond to $\xi=0.01,1$, and 5, respectively. Filled circles and squares are the real and imaginary parts of the spectrum $\chi_{D 2}(\omega)$ of the normalized exponential relaxation function with the relaxation time $\tau_{2}$ from Eq. (3.18).

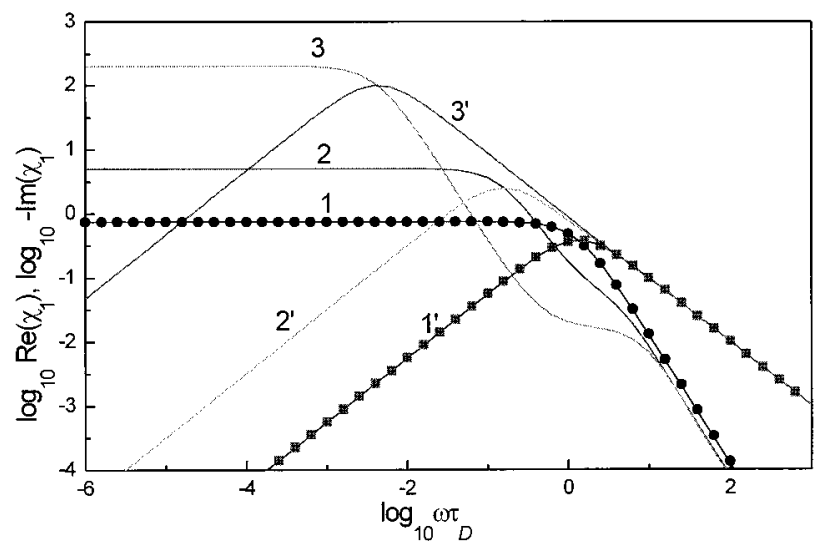

FIG. 7. Real (curves $1,2,3$ ) and imaginary (curves $1^{\prime}, 2^{\prime}, 3^{\prime}$ ) parts of the spectrum $\chi_{1}(\omega)$ of the normalized relaxation function at $\xi=2$. Curves $1,1^{\prime}, 2,2^{\prime}$, and $3,3^{\prime}$ correspond to $\sigma=0.01,5$, and 10 , respectively. Filled circles and squares are the real and imaginary parts of the spectrum $\chi_{D 1}(\omega)$ of the normalized exponential relaxation function with the relaxation time $\tau_{1}$ from Eq. (4.14).

Thus, in the context of the rotational diffusion model the switch-on nonlinear dielectric relaxation and dynamic Kerr effect responses of an ensemble of noninteracting polar molecules can be evaluated from exact analytic equations. The range of applicability of the results obtained is $\omega \tau_{n} \approx 1$, as inertial effects are ignored in our model. In order to take into account inertial effects in the theory one should consider the inertial term in Eq. (2.2). However, the calculation will now become very much more complicated [7].

The analytical treatment of the nonlinear dielectric relaxation and the transient electric birefrigence presented in the paper can also be applied to other problems considered by Watanabe and Morita [6]. In particular, our approach can be extended to a homogeneous electric field suddenly applied to a system in which a Maxwell-Boltzmann distribution of particle orientation has been established by another homogeneous electric field, and to a homogeneous electric field suddenly reversed or rapidly rotating. For all these problems the hierarchy of differential-recurrence relations for averaged

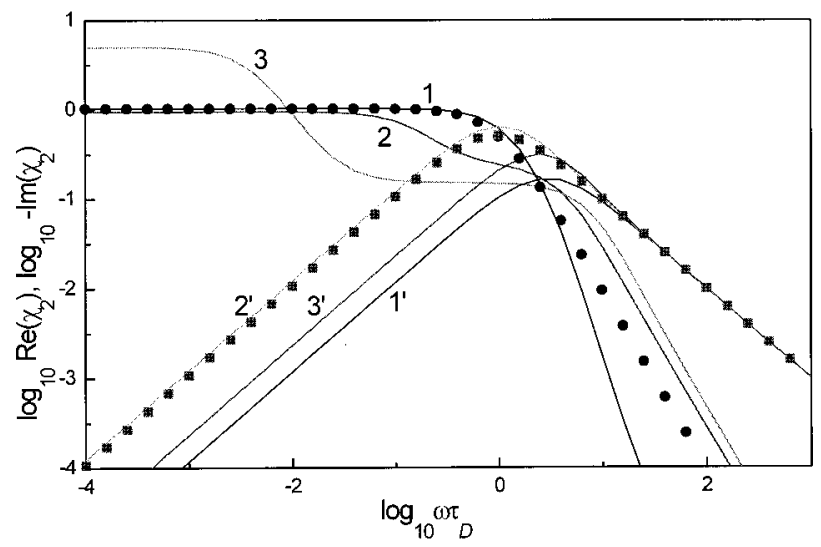

FIG. 8. Real (curves 1,2,3) and imaginary (curves $1^{\prime}, 2^{\prime}, 3^{\prime}$ ) parts of the spectrum $\chi_{2}(\omega)$ of the normalized relaxation function at $\xi=2$. Curves $1,1^{\prime}, 2,2^{\prime}$, and $3,3^{\prime}$ correspond to $\sigma=0.01,5$, and 10 , respectively. Filled circles and squares are the real and imaginary parts of the spectrum $\chi_{D 2}(\omega)$ of the normalized exponential relaxation function with the relaxation time $\tau_{2}$ from Eq. (4.15). 
spherical harmonics (2.22) can always be reduced to the three-term matrix equation (5.1) the solution of which is given by Eq. (5.3). All that remains is to evaluate the initial value vectors $\mathbf{C}_{n}(0)$.

Our approach can be used for the evaluation of the nonlinear response of analogous physical systems. In particular, the results presented in Sec. V can also be applied with a small modification to the calculation of the nonlinear dielectric response of nematic liquid crystals and to the appropriate nonlinear magnetic response of an assembly of single domain ferromagnetic particles. In the both cases the longitudinal relaxation (dielectric and magnetic, respectively) of these systems is governed by Eq. (5.2) with a different interpretation for the parameters $\xi$ and $\sigma$ (for details see Refs. [7, $14,20,22,25])$. Again, the solution for the Laplace transforms of appropriate relaxation functions describing the nonlinear response of these systems is given by Eq. (5.3) with the appropriate initial value vectors $\mathbf{C}_{n}(0)$.

\section{ACKNOWLEDGMENTS}

One of us (Y.P.K.) thanks the French Ministry of High Education and Research for financial support (high level grant, PECO-CEI). W.T.C. acknowledges the financial support of the Forbairt Research Collaboration Fund and the French Foreign Office. The partial support of this work by the Russian Foundation for Basic Research (Grant No. 9602-16762-a) is also gratefully acknowledged.

\section{APPENDIX: DERIVATION OF EQ. (2.13)}

Noting that the rule for changing of variables in Stratonovich differential equations is the same as in ordinary analysis [18], we obtain from Eq. (2.10) a stochastic equation for an arbitrary differentiable function $f(\{\boldsymbol{\xi}(t)\})$ :

$$
\begin{aligned}
\frac{d}{d t} f(\{\boldsymbol{\xi}(t)\})= & h_{i}(\{\boldsymbol{\xi}(t)\}, t) \frac{\partial}{\partial \xi_{i}} f(\{\boldsymbol{\xi}(t)\}) \\
& +g_{i j}(\{\boldsymbol{\xi}(t)\}, t) \frac{\partial}{\partial \xi_{i}} f(\{\boldsymbol{\xi}(t)\}) \Gamma_{j}(t) .
\end{aligned}
$$

We remark that from the mathematical point of view the stochastic differential equation (A1) [just as Eq. (2.10)] with the $\delta$-correlated Langevin forces $\Gamma_{i}(t)$ is not completely defined $[7,18]$. The most satisfactory interpretation of Eq. (2.10) and (A1) is as the stochastic integral equation [5,7]

$$
\begin{aligned}
\xi_{i}(t+\tau)= & x_{i}+\int_{0}^{\tau} h_{i}\left(\left\{\boldsymbol{\xi}\left(t+t^{\prime}\right)\right\}, t+t^{\prime}\right) d t^{\prime} \\
& +\sqrt{D} \int_{0}^{\tau} g_{i j}\left(\left\{\boldsymbol{\xi}\left(t+t^{\prime}\right)\right\}, t+t^{\prime}\right) d w_{j}\left(t^{\prime}\right)
\end{aligned}
$$

$$
\begin{aligned}
f(\{\boldsymbol{\xi}(t+\tau)\})= & f(\{\mathbf{x}\})+\int_{0}^{\tau} h_{i}\left(\left\{\xi \boldsymbol{\xi}\left(t+t^{\prime}\right)\right\}, t+t^{\prime}\right) \\
& \times \frac{\partial}{\partial \xi_{i}} f\left(\left\{\boldsymbol{\xi}\left(t+t^{\prime}\right)\right\}\right) d t^{\prime} \\
& +\sqrt{D} \int_{0}^{\tau} g_{i j}\left(\left\{\boldsymbol{\xi}\left(t+t^{\prime}\right)\right\}, t+t^{\prime}\right) \\
& \times \frac{\partial}{\partial \xi_{i}} f\left(\left\{\boldsymbol{\xi}\left(t+t^{\prime}\right)\right\}\right) d w_{j}\left(t^{\prime}\right),
\end{aligned}
$$

where

$$
w_{j}(\tau)=\frac{1}{\sqrt{D}} \int_{t}^{t+\tau} \Gamma_{j}\left(t^{\prime}\right) d t^{\prime},
$$

the Stratonovich stochastic integral of a function $F$ is defined as [5]

$$
\begin{aligned}
& \int_{t}^{t+\tau} F\left(\left\{\boldsymbol{\xi}\left(t^{\prime}\right)\right\}, t^{\prime}\right) d w_{j}\left(t^{\prime}\right) \\
&=\lim _{\Delta \rightarrow 0} \sum_{i=0}^{n-1} F\left(\left\{\frac{\boldsymbol{\xi}\left(t_{i}\right)+\boldsymbol{\xi}\left(t_{i+1}\right)}{2}\right\}, \frac{t_{i}+t_{i+1}}{2}\right) \\
& \quad \times\left[w_{j}\left(t_{i+1}\right)-w_{j}\left(t_{i}\right)\right], \\
& \Delta= \max \left(t_{i+1}-t_{i}\right), \quad t=t_{0}<t_{1}<\ldots<t_{n}=t+\tau,
\end{aligned}
$$

$\{\mathbf{w}(t)\}=\left\{w_{1}(t), w_{2}(t), \ldots, w_{N}(t)\right\}$ is the Wiener process in $R^{N}$ with the following properties:

$$
\overline{w_{j}(t)}=0, \quad \overline{\int_{0}^{\tau} w_{i}(t) d w_{j}(t)}=\tau \delta_{i j} .
$$

On supposing that the integrands in Eqs. (A2) and (A3) can be expanded in Taylor series, we obtain

$$
\begin{aligned}
\xi_{i}(t+\tau)= & x_{i}+\int_{0}^{\tau} h_{i}\left(\{\mathbf{x}\}, t+t^{\prime}\right) d t^{\prime} \\
& +\int_{0}^{\tau}\left[\xi_{k}\left(t+t^{\prime}\right)-x_{k}\right] \frac{\partial}{\partial x_{k}} h_{i}\left(\{\mathbf{x}\}, t+t^{\prime}\right) d t^{\prime} \\
& +\sqrt{D} \int_{0}^{\tau} g_{i j}\left(\{\mathbf{x}\}, t+t^{\prime}\right) d w_{j}\left(t^{\prime}\right) \\
& +\sqrt{D} \int_{0}^{\tau}\left[\xi_{k}\left(t+t^{\prime}\right)-x_{k}\right] \\
& \times \frac{\partial}{\partial x_{k}} g_{i j}\left(\{\mathbf{x}\}, t+t^{\prime}\right) d w_{j}\left(t^{\prime}\right),
\end{aligned}
$$




$$
\begin{aligned}
f(\{\boldsymbol{\xi}(t+\tau)\})= & f(\{\mathbf{x}\})+\int_{0}^{\tau} h_{i}\left(\{\mathbf{x}\}, t+t^{\prime}\right) \frac{\partial}{\partial x_{i}} f(\{\mathbf{x}\}) d t^{\prime} \\
& +\int_{0}^{\tau}\left[\xi_{k}\left(t+t^{\prime}\right)-x_{k}\right] \frac{\partial}{\partial x_{k}}\left[h_{i}\left(\{\mathbf{x}\}, t+t^{\prime}\right)\right. \\
& \left.\times \frac{\partial}{\partial x_{i}} f(\{\mathbf{x}\})\right] d t^{\prime}+\sqrt{D} \int_{0}^{\tau} g_{i j}\left(\{\mathbf{x}\}, t+t^{\prime}\right) \\
& \times \frac{\partial}{\partial x_{i}} f(\{\mathbf{x}\}) d w_{j}\left(t^{\prime}\right) \\
& +\sqrt{D} \int_{0}^{\tau}\left[\xi_{k}\left(t+t^{\prime}\right)-x_{k}\right] \\
& \times \frac{\partial}{\partial x_{k}}\left[g_{i j}\left(\left\{\mathbf{x}_{\}}, t+t^{\prime}\right) \frac{\partial}{\partial x_{i}} f(\{\mathbf{x}\})\right] d w_{j}\left(t^{\prime}\right)\right. \\
& +\cdots
\end{aligned}
$$

Following ([5], pp. 51-53) [namely, substituting $\xi_{i}\left(t+t^{\prime}\right)$ from Eq. (A6) into Eq. (A7), then integrating and averaging

[1] J. R. McConnell, Rotational Brownian Motion and Dielectric Theory (Academic, NY, 1981).

[2] J-L. Déjardin, Dynamic Kerr Effect (World Scientific, Singapore, 1995).

[3] H. Benoit, Ann. Phys. (N.Y.) 6, 561 (1951).

[4] A. Morita, Phys. Rev. A 34, 1499 (1986).

[5] H. Risken, The Fokker-Planck Equation (Springer, Berlin, 1984).

[6] H. Watanabe and A. Morita, Adv. Chem. Phys. 56, 255 (1984).

[7] W. T. Coffey, Yu. P. Kalmykov, and J. T. Waldron, The Langevin Equation (World Scientific, Singapore, 1996).

[8] M. S. Beevers, J. Crossley, D. C. Garrington, G. Williams, J. Chem. Soc. Faraday Trans. 2, 72, 1482 (1976).

[9] W. T. Coffey, Yu. P. Kalmykov, E. S. Massawe, and J. T. Waldron, J. Chem. Phys. 99, 4011 (1993).

[10] W. T. Coffey, D. S. F. Crothers, Yu. P. Kalmykov, E. S. Massawe, and J. T. Waldron, Phys. Rev. E 49, 1869 (1994).

[11] W. T. Coffey, D. S. F. Crothers, Yu. P. Kalmykov, and J. T. Waldron, Physica A 213, 551 (1994).

[12] J. T. Waldron, Yu. P. Kalmykov, and W. T. Coffey, Phys. Rev. E 49, 3976 (1994).

[13] W. T. Coffey, Yu. P. Kalmykov, and J. T. Waldron, Physica A 208, 462 (1994).

[14] W. T. Coffey, D. S. F. Crothers, Yu. P. Kalmykov, and J. T. Waldron, Phys. Rev. B 51, 15947 (1995). the equation so obtained taking account of the properties (A5) and retaining only the terms proportional to $\tau$ ], we obtain

$$
\begin{aligned}
\frac{\overline{f(\{\boldsymbol{\xi}(t+\tau)\})}-f(\{\mathbf{x}\})}{\tau}= & h_{i}\left(\{\mathbf{x}\}, t+\tau \Theta_{i i i}^{(1)}\right) \frac{\partial}{\partial x_{i}} f(\{\mathbf{x}\}) \\
& +D g_{k j}\left(\{\mathbf{x}\}, t+\tau \Theta_{i j k}^{(2)} \Theta_{i j k}^{(3)}\right) \frac{\partial}{\partial x_{k}} \\
& \times\left[g_{i j}\left(\{\mathbf{x}\}, t+\tau \Theta_{i j k}^{(3)}\right) \frac{\partial}{\partial x_{i}} f(\{\mathbf{x}\})\right] \\
& +O(\tau),
\end{aligned}
$$

where $\Theta_{i j k}^{(n)}$ are constants $\left(0 \leqslant \Theta_{i j k}^{(n)} \leqslant 1\right)$. Taking the limit $\tau \rightarrow 0$ in Eq. (A8), we have Eq. (2.13).
[15] J-L. Déjardin, P. Blaise, and W. T. Coffey, Phys. Rev. E 54, 852 (1996).

[16] Yu. P. Kalmykov, J. Mol. Liq. 69, 117 (1996).

[17] R. L. Stratonovich, Conditional Markov Processes and Their Applications in the Theory of Optimal Control (MGU Publishers, Moscow, 1968).

[18] C. W. Gardiner, Handbook of Stochastic Methods (Springer, Berlin, 1982).

[19] Handbook of Mathematical Functions, edited by M. Abramowitz and I. Stegun (Dover, New York, 1965).

[20] A. J. Martin, G. Meier, and A. Saupe, Symp. Faraday Soc. 5, 119 (1971)

[21] W. F. Brown, Jr., Phys. Rev. 130, 1677 (1963).

[22] Yu. L. Raikher and M. I. Shliomis, Adv. Chem. Phys. 87, 595 (1994).

[23] M. A. Martsenyuk, Yu. L. Raikher, and M. I. Shliomis, Zh. Eksp. Teor. Fiz. 65, 834 (1973) [Sov. Phys. JETP 38, 413 (1974)].

[24] Yu. L. Raikher and M. I. Shliomis, Zh. Eksp. Teor. Fiz. 67, 1060 (1974) [Sov. Phys. JETP 40, 526 (1974)].

[25] W. T. Coffey, P. J. Cregg, and Yu. P. Kalmykov, Advances in Chemical Physics, edited by I. Prigogine and S. A. Rice (Wiley, New York, 1993), Vol. 83, pp. 263-464.

[26] A. P. Prudnikov, Yu. A. Brychkov, and O. I. Marichev, Integrals and Series. Additional Chapters (Science, Moscow, 1986) p. 476.

[27] W. M. Tolles, J. Appl. Phys. 46, 991 (1975). 